\title{
Killer Cell Immunoglobulin-Like Receptor Genes Polymorphisms in Macedonian Patients with Haematological Malignancies
}

\author{
Aleksandar Petlichkovski ${ }^{1}$, Zlate Stojanoski², Dragan Cibrev $^{1}$, Mirko Spiroski ${ }^{1 *}$ \\ IInstitute of Immunobiology and Human Genetics, Faculty of Medicine, Ss Cyril and Methodius University, Skopje, Republic of Macedonia; \\ ${ }^{2}$ University Clinic of Haematological Diseases, Faculty of Medicine, Ss Cyril and Methodius University, Skopje, Republic of Macedonia
}

\begin{abstract}
Citation: Petlichkovski A, Stojanoski Z, Cibrev D, Spiroski M. Killer Cell Immunoglobulin-Like Receptor Genes Polymorphisms in Macedonian Patients with Haematological Malignancies. Maced J Med Sci. 2013 Mar 15; O(1):24-30. htp. /dx.doi.org/10.3889/M JMS.1857. 5773.2012.0271.

Key words: Killer immunoglobulin-like receptor (KIR) gene polymorphism; SSP KIR genotyping; Haematological malignancies; Republic of Macedonia.

Correspondence: Mirko Spiroski, MD, PhD. Institute of Immunobiology and Human Genetics, Faculty of Medicine, Ss Cyril and Methodius Faculty of Medicine, Ss Cyril and Methodius
University of Skopje, 1109 Skopje, PO Box 60 , Republic of Macedonia. Tel.: +389-2-3110556 Fax: +389-2-3110558.

E-mail: mspiroski@yahoo.com

Received: 29-Sep-2012; Revised: 25-Nov-2012; Accepted: 17-Dec-2012; Online first: 19-Dec-2012 Copyright: @ 2012 Petlichkovski A. This is an open-access article distributed under the terms of the Creative Commons Attribution License, which permits unrestricted use, distribution, and reproduction in any medium, provided the original author and source are credited.

Competing Interests: The author have declared that no competing interests exist.
\end{abstract}

\section{Abstract}

Aim: The aim of this study was to examine the gene frequencies of $16 \mathrm{KIR}$ genes and pseudogenes (KIR2DL1, KIR2DL2, KIR2DL3, KIR2DL4, KIR2DL5, KIR3DL1, KIR3DL2, KIR3DL3, KIR2DS1, KIR2DS2, KIR2DS3, KIR2DS4, KIR2DS5, KIR3DS1, KIR2DP1, and KIR3DP1) and KIR genotypes in Macedonian patients with haematological malignancies, to compare them with the corresponding frequencies of healthy Macedonians and to analyse eventual association of specific genes or genotypes with the studied haematological diseases.

Material and Methods: The study included 63 patients of which 40 had acute myeloblastic leukaemia (63.5\%), 11 (17.5\%) had chronic myeloid leukaemia, 8 (12.7\%) acute lymphoblastic leukaemia, 2 (3.17\%) non-Hodgkin Lymphoma, 1 (1.59\%) aplastic anemia, and 1 (1.59\%) chronic lymphocytic leukaemia.

Results: Comparison of KIR gene frequencies between the 63 patients and healthy Macedonians reveals statistically significant difference for KIR3DL2 ( $F=1$ in the control group, and 0.95 in the patients group, $\mathrm{p}=0.001$ ). Another statistically significant difference was observed for the frequency of $\mathrm{B} \times 3$ and $\mathrm{B} \times 439$ genotypes both found more often in patients group $(\mathrm{P}=0.017$ and $\mathrm{P}=0.009$, respectively).

Conclusion: Further analysis, involving larger series of patients and targeted at the ligands of the KIRs are needed in order to determine a certain KIR gene and/or genotype as either predisposing, or protecting factor for haematological malignancy in patients from Republic of Macedonia.

\section{Introduction}

The Natural killer (NK) cells are a subset of lymphocytes comprising around $10 \%$ of total lymphocytes in peripheral blood [1]. Early recognition and killing of virally infected cells and tumour cells are among the most important roles of the NK cells in the innate immunity $[2,3]$. The NK cell activity is regulated in activating or inhibiting manner, through specific receptors present on their surface, called killer cell immunoglobulin like receptors (KIRs). The KIRs are encoded by the KIR gene complex, located on chromosome 19, containing a family of polymorphic and highly homologous members (14 genes and 2 pseudogenes), which can be activating or inhibitory [4]. Based on the gene content, two basic KIR haplotypes have been defined, termed $A$ and $B$. The A haplotype contains a single activating gene, KIR2DS4, which is often present in a deleted (non-expressed) 
form, while the $B$ haplotype is more variable and contains many activating genes $[5,6]$.

The different KIR haplotypes vary in the number and the type of genes present, but the genes KIR3DL3, KIR3DP1, KIR2DL4 and KIR3DL2 are present on virtually all haplotypes and have therefore been termed framework genes [7]. Population studies performed over the last two decades have revealed extensive diversity at the KIR gene locus, which derives from both, its polygenic and multi-allelic polymorphism, whereas on the basis of gene content, haplotype $\mathrm{B}$ displays a much greater variety of subtypes [6, 8].

The most important for the triggering or inhibition of the NK cells is the balance between the signals generated by the KIRs and their ligands, the HLA molecules $[9,10]$. Several recent studies have shown lowered expression of HLA class I molecules in patients with leukaemia [11-13]. It is believed that the down regulation of HLA class I molecules in leukaemia cells releases the inhibitory influence on NK cells, thus permitting the NK cells to activate and lyse the altered target cells. Hypothesizing that NK cells play a major role in the innate immune surveillance of leukaemia cells, several groups have analyzed the KIR gene content in leukaemia patients, but have reported contradictory results [14-17].

The aim of this study was to examine the gene frequencies of $16 \mathrm{KIR}$ genes and pseudogenes (KIR2DL1, KIR2DL2, KIR2DL3, KIR2DL4, KIR2DL5, KIR3DL1, KIR3DL2, KIR3DL3, KIR2DS1, KIR2DS2, KIR2DS3, KIR2DS4, KIR2DS5, KIR3DS1, KIR2DP1, and $K I R 3 D P 1)$ and $K I R$ genotypes in Macedonian patients with haematological malignancies, to compare them with the corresponding frequencies of healthy Macedonians and to analyse eventual association of specific genes or genotypes with the studied haematological diseases.

To our knowledge, this is the first study of the association of $K I R$ genes with haematological malignant diseases in the Republic of Macedonia.

\section{Material and Methods}

\section{Population samples}

The study was performed on 63 patients with haematological malignancies hospitalized and treated at the Haematology University Clinic in Skopje, between June, 2001 and September 2009. Distribution according to diagnosis was: acute myeloblastic leukemia 40 (63.5\%), chronic myeloid leukemia 11 (17.5\%), acute lymphoblastic leukemia 8 (12.7\%), non-Hodgkin Lymphoma 2 (3.17\%), aplastic anemia 1 (1.59\%), and chronic lymphocytic leukemia 1 (1.59\%).

\section{KIR genotyping}

After signing of written consent, genomic DNA was extracted from the peripheral blood leukocytes using standard phenol/chloroform procedure, described elsewhere [18], and stored in the Macedonian Human DNA Bank (hDNAMKD) [19] until processing. For KIR genotyping, commercially available PEL-FREEZ KIR genotyping SSP kit (Dynal Biotech, Brown Deer, WI) was used. It is a PCR-based method (using sequencespecific priming approach) designed to detect the presence or absence of 16 KIR genes. The presence of each KIR gene was determined by the presence of a band of DNA of the expected size. All PCRs contained an internal positive control consisting of an additional pair of primers specific for the growth hormone $(\mathrm{GH})$ gene and a negative control. Individuals were determined

Table 1: Comparison of the observed and estimated KIR gene frequencies for Macedonian patients with haematological malignancies $(\mathrm{N}=63)$ and healthy Macedonians $(\mathrm{N}=214)$.

\begin{tabular}{|c|c|c|c|c|c|c|c|c|c|c|c|c|c|c|c|c|}
\hline & \multicolumn{16}{|c|}{ Frequencies for KIR genes in Macedonian patients with haematological malignancies and Macedonians } \\
\hline & \multicolumn{2}{|c|}{ Pseudogenes } & \multicolumn{8}{|c|}{ Inhibitory KIR } & \multicolumn{6}{|c|}{ Non inhibitory KIR } \\
\hline & $\begin{array}{c}K I R \\
2 D P 1\end{array}$ & $\begin{array}{c}K I R \\
3 D P 1\end{array}$ & $\begin{array}{c}K I R \\
2 D L 1\end{array}$ & $\begin{array}{c}\text { KIR } \\
2 D L 2 \\
\end{array}$ & $\begin{array}{c}K I R \\
2 D L 3 \\
\end{array}$ & $\begin{array}{c}\text { KIR } \\
2 D L 4\end{array}$ & $\begin{array}{c}K I R \\
2 D L 5\end{array}$ & $\begin{array}{c}K I R \\
3 D L 1\end{array}$ & $\begin{array}{c}K I R \\
3 D L 2\end{array}$ & $\begin{array}{c}K I R \\
3 D L 3 \\
\end{array}$ & $\begin{array}{c}K I R \\
2 D S 1\end{array}$ & $\begin{array}{c}K I R \\
2 D S 2\end{array}$ & $\begin{array}{c}K I R \\
2 D S 3\end{array}$ & $\begin{array}{c}K I R \\
2 D S 4\end{array}$ & $\begin{array}{c}\text { KIR } \\
2 D S 5 \\
\end{array}$ & $\begin{array}{c}K I R \\
3 D S 1\end{array}$ \\
\hline Malignancies (N) & 61 & 63 & 61 & 35 & 58 & 63 & 19 & 60 & 60 & 63 & 29 & 35 & 21 & 62 & 21 & 30 \\
\hline Malignancies (F) & 0.968 & 1 & 0.968 & 0.556 & 0.921 & 1 & 0.302 & 0.952 & 0.952 & 1 & 0.651 & 0.556 & 0.333 & 0.984 & 0.333 & 0.397 \\
\hline Malignancies (GF) & 0.821 & 1 & 0.821 & 0.334 & 0.717 & 1 & 0.165 & 0.781 & 0.781 & 1 & 0.409 & 0.334 & 0.183 & 0.874 & 0.183 & 0.223 \\
\hline Macedonians (N) & 210 & 214 & 201 & 126 & 192 & 214 & 89 & 201 & 214 & 214 & 103 & 122 & 77 & 201 & 64 & 84 \\
\hline Macedonians (F) & 0.980 & 1 & 0.940 & 0.590 & 0.897 & 1 & 0.415 & 0.940 & 1 & 1 & 0.481 & 0.570 & 0.360 & 0.940 & 0.300 & 0.392 \\
\hline Macedonians (GF) & 0.870 & 1 & 0.760 & 0.360 & 0.690 & 1 & 0.230 & 0.800 & 1 & 1 & 0.280 & 0.350 & 0.180 & 0.800 & 0.170 & 0.220 \\
\hline Pearson's p & 0.532 & $\&$ & 0.371 & 0.638 & 0.581 & $\&$ & 0.102 & 0.695 & 0.001 & $\&$ & 0.769 & 0.838 & 0.699 & 0.153 & 0.604 & 0.236 \\
\hline Risk Ratio & 0.99 & 1 & 1.03 & 0.94 & 1.03 & 1 & 0.73 & 1.01 & $\&$ & 1 & 0.96 & 0.97 & 0.93 & 1.05 & 1.11 & 1.21 \\
\hline $95 \% \mathrm{Cl}$ & $\&$ & $\&$ & $\&$ & $\begin{array}{c}0.737- \\
1.209\end{array}$ & $\begin{array}{c}0.942- \\
1.118\end{array}$ & $\&$ & $\begin{array}{c}0.482- \\
1.091\end{array}$ & $\begin{array}{r}0.950 \\
1.082\end{array}$ & $\&$ & $\&$ & $\begin{array}{c}0.708- \\
1.293\end{array}$ & $\begin{array}{c}0.759 \\
1.251\end{array}$ & $\begin{array}{l}0.626 \\
1.371\end{array}$ & $\begin{array}{l}1.004- \\
1.097\end{array}$ & $\begin{array}{c}0.743- \\
1.671\end{array}$ & $\begin{array}{l}0.892- \\
1.651\end{array}$ \\
\hline
\end{tabular}

$\mathrm{N}$, number of individuals; $\mathrm{F}$, observed frequency was obtained by direct counting; GF, gene frequencies were calculated using the formula GF=1-Ö(1-F); p, statistical significance; \&, cannot be calculated because expected $<5, \mathrm{c} 2$ test; RR, Risk Ratio; $\mathrm{Cl}$, confidence interval. 
negative for a particular KIR gene when a band of the GH gene. We have used external quality control expected size was absent in the presence of a band for consisting of cell lines from Immunogenetics and

Table 2: KIR gene content of Macedonian patients with haematological malignancies $(\mathrm{N}=63)$.

\begin{tabular}{|c|c|c|c|c|c|c|c|c|c|c|c|c|c|c|c|c|c|}
\hline Patient ID & $\mathrm{Dg}$ & $\begin{array}{c}K I R \\
3 D L 1\end{array}$ & $\begin{array}{c}K I R \\
2 D L 1\end{array}$ & $\begin{array}{c}K I R \\
2 D L 3\end{array}$ & $\begin{array}{c}K I R \\
2 D S 4\end{array}$ & $\begin{array}{c}K I R \\
2 D L 2\end{array}$ & $\begin{array}{c}K I R \\
2 D L 5\end{array}$ & $\begin{array}{c}K I R \\
3 D S 1\end{array}$ & $\begin{array}{c}K I R \\
2 D S 1\end{array}$ & $\begin{array}{c}\text { KIR } \\
2 D S 2\end{array}$ & $\begin{array}{c}K I R \\
2 D S 3\end{array}$ & $\begin{array}{c}K I R \\
2 D S 5\end{array}$ & $\begin{array}{c}K I R \\
2 D L 4\end{array}$ & $\begin{array}{c}K I R \\
3 D L 2\end{array}$ & $\begin{array}{c}K I R \\
3 D L 3\end{array}$ & $\begin{array}{c}K I R \\
2 D P 1\end{array}$ & $\begin{array}{c}K I R \\
3 D P 1\end{array}$ \\
\hline PTH001 & ALL & 1 & 1 & 1 & 1 & 1 & 0 & 0 & 0 & 1 & 0 & 0 & 1 & 1 & 1 & 1 & 1 \\
\hline PTH009 & CML & 0 & 1 & 1 & 1 & 0 & 1 & 1 & 1 & 1 & 1 & 0 & 1 & 1 & 1 & 1 & 1 \\
\hline PTH014 & AML & 0 & 1 & 1 & 1 & 0 & 0 & 0 & 1 & 0 & 0 & 0 & 1 & 0 & 1 & 1 & 1 \\
\hline PTH018 & ALL & 1 & 1 & 1 & 1 & 0 & 0 & 0 & 0 & 0 & 0 & 0 & 1 & 1 & 1 & 1 & 1 \\
\hline PTH026 & AML & 1 & 1 & 1 & 1 & 1 & 0 & 0 & 1 & 1 & 1 & 0 & 1 & 1 & 1 & 1 & 1 \\
\hline PTH031 & CML & 1 & 1 & 1 & 1 & 0 & 0 & 0 & 0 & 0 & 0 & 0 & 1 & 1 & 1 & 1 & 1 \\
\hline PTH037 & AML & 1 & 1 & 1 & 1 & 1 & 0 & 1 & 1 & 1 & 1 & 1 & 1 & 1 & 1 & 1 & 1 \\
\hline PTH042 & AML & 1 & 1 & 1 & 1 & 0 & 0 & 0 & 1 & 0 & 0 & 0 & 1 & 1 & 1 & 1 & 1 \\
\hline PTH057 & $\mathrm{NHL}$ & 1 & 1 & 1 & 1 & 1 & 0 & 1 & 1 & 1 & 1 & 1 & 1 & 1 & 1 & 1 & 1 \\
\hline PTH074 & CML & 1 & 1 & 0 & 1 & 1 & 0 & 1 & 0 & 1 & 1 & 0 & 1 & 1 & 1 & 1 & 1 \\
\hline PTH102 & AML & 1 & 1 & 1 & 1 & 1 & 1 & 1 & 1 & 1 & 1 & 0 & 1 & 1 & 1 & 1 & 1 \\
\hline PTH106 & CLL & 1 & 1 & 1 & 1 & 1 & 1 & 1 & 1 & 1 & 0 & 1 & 1 & 1 & 1 & 1 & 1 \\
\hline PTH1 12 & AML & 1 & 1 & 0 & 1 & 1 & 0 & 0 & 1 & 1 & 1 & 0 & 1 & 1 & 1 & 1 & 1 \\
\hline PTH123 & AML & 1 & 1 & 1 & 1 & 0 & 0 & 0 & 0 & 0 & 0 & 0 & 1 & 1 & 1 & 1 & 1 \\
\hline PTH135 & CML & 1 & 1 & 1 & 1 & 0 & 0 & 0 & 1 & 0 & 0 & 0 & 1 & 1 & 1 & 1 & 1 \\
\hline PTH156 & AML & 1 & 1 & 1 & 1 & 1 & 1 & 1 & 1 & 1 & 1 & 1 & 1 & 1 & 1 & 1 & 1 \\
\hline PTH168 & CML & 1 & 1 & 1 & 1 & 0 & 0 & 0 & 0 & 0 & 0 & 0 & 1 & 1 & 1 & 1 & 1 \\
\hline PTH176 & AML & 1 & 1 & 1 & 1 & 0 & 1 & 1 & 1 & 0 & 0 & 1 & 1 & 1 & 1 & 1 & 1 \\
\hline PTH179 & AML & 1 & 1 & 1 & 1 & 0 & 0 & 0 & 1 & 0 & 0 & 0 & 1 & 1 & 1 & 1 & 1 \\
\hline PTH199 & $\mathrm{NHL}$ & 1 & 1 & 1 & 1 & 1 & 0 & 0 & 0 & 1 & 0 & 0 & 1 & 1 & 1 & 1 & 1 \\
\hline PTH195 & AML & 1 & 0 & 0 & 1 & 1 & 0 & 1 & 1 & 1 & 0 & 1 & 1 & 1 & 1 & 0 & 1 \\
\hline PTH235 & LA & 1 & 0 & 0 & 1 & 1 & 0 & 1 & 1 & 1 & 0 & 1 & 1 & 1 & 1 & 0 & 1 \\
\hline PTH265 & AML & 1 & 1 & 1 & 1 & 1 & 0 & 1 & 1 & 1 & 1 & 1 & 1 & 1 & 1 & 1 & 1 \\
\hline PTH278 & AML & 1 & 1 & 1 & 1 & 1 & 1 & 0 & 0 & 1 & 1 & 0 & 1 & 1 & 1 & 1 & 1 \\
\hline PTH294 & AML & 1 & 1 & 1 & 1 & 0 & 0 & 0 & 0 & 1 & 0 & 0 & 1 & 1 & 1 & 1 & 1 \\
\hline PTH299 & ALL & 1 & 1 & 1 & 1 & 0 & 0 & 0 & 0 & 0 & 0 & 0 & 1 & 1 & 1 & 1 & 1 \\
\hline PTH306 & AML & 1 & 1 & 1 & 1 & 0 & 0 & 0 & 0 & 0 & 0 & 0 & 1 & 1 & 1 & 1 & 1 \\
\hline PTH308 & CML & 1 & 1 & 1 & 1 & 1 & 0 & 0 & 1 & 1 & 1 & 0 & 1 & 1 & 1 & 1 & 1 \\
\hline PTH326 & AML & 1 & 1 & 1 & 1 & 0 & 0 & 0 & 0 & 0 & 0 & 0 & 1 & 1 & 1 & 1 & 1 \\
\hline PTH329 & AML & 1 & 1 & 1 & 1 & 1 & 1 & 0 & 1 & 1 & 1 & 0 & 1 & 1 & 1 & 1 & 1 \\
\hline PTH349 & ALL & 1 & 1 & 1 & 1 & 1 & 1 & 0 & 1 & 1 & 1 & 0 & 1 & 1 & 1 & 1 & 1 \\
\hline PTH354 & A apl. & 1 & 1 & 1 & 1 & 1 & 0 & 0 & 0 & 1 & 0 & 0 & 1 & 1 & 1 & 1 & 1 \\
\hline PTH358 & AML & 1 & 1 & 1 & 1 & 1 & 1 & 1 & 1 & 1 & 0 & 1 & 1 & 1 & 1 & 1 & 1 \\
\hline PTH365 & ALL & 1 & 1 & 1 & 1 & 1 & 0 & 1 & 1 & 1 & 1 & 0 & 1 & 1 & 1 & 1 & 1 \\
\hline PTH4 17 & AML & 1 & 1 & 1 & 1 & 1 & 0 & 0 & 1 & 0 & 0 & 0 & 1 & 1 & 1 & 1 & 1 \\
\hline PTH455 & AML & 1 & 1 & 1 & 1 & 0 & 0 & 0 & 0 & 0 & 0 & 0 & 1 & 1 & 1 & 1 & 1 \\
\hline PTH472 & AML & 1 & 1 & 1 & 1 & 0 & 0 & 0 & 0 & 0 & 0 & 0 & 1 & 1 & 1 & 1 & 1 \\
\hline PTH486 & AML & 1 & 1 & 1 & 1 & 0 & 0 & 1 & 1 & 0 & 0 & 1 & 1 & 1 & 1 & 1 & 1 \\
\hline PTH490 & AML & 1 & 1 & 1 & 1 & 1 & 0 & 1 & 1 & 1 & 0 & 1 & 1 & 1 & 1 & 1 & 1 \\
\hline PTH 493 & ALL & 1 & 1 & 1 & 1 & 1 & 0 & 0 & 1 & 1 & 1 & 0 & 1 & 1 & 1 & 1 & 1 \\
\hline PTH526 & AML & 1 & 1 & 1 & 1 & 1 & 1 & 1 & 1 & 1 & 0 & 1 & 1 & 1 & 1 & 1 & 1 \\
\hline PTH530 & ALL & 1 & 1 & 1 & 1 & 0 & 0 & 1 & 1 & 0 & 0 & 1 & 1 & 1 & 1 & 1 & 1 \\
\hline PTH538 & AML & 1 & 1 & 1 & 1 & 1 & 1 & 1 & 1 & 1 & 0 & 1 & 1 & 1 & 1 & 1 & 1 \\
\hline PTH541 & CML & 1 & 1 & 1 & 1 & 1 & 1 & 0 & 1 & 1 & 1 & 0 & 1 & 1 & 1 & 1 & 1 \\
\hline PTH544 & AML & 1 & 1 & 1 & 1 & 1 & 1 & 1 & 1 & 1 & 1 & 1 & 1 & 1 & 1 & 1 & 1 \\
\hline PTH550 & AML & 1 & 1 & 1 & 1 & 0 & 0 & 0 & 1 & 0 & 0 & 0 & 1 & 1 & 1 & 1 & 1 \\
\hline PTH554 & AML & 1 & 1 & 1 & 1 & 1 & 1 & 1 & 1 & 1 & 0 & 1 & 1 & 1 & 1 & 1 & 1 \\
\hline PTH595 & AML & 1 & 1 & 1 & 1 & 1 & 1 & 1 & 1 & 1 & 1 & 1 & 1 & 1 & 1 & 1 & 1 \\
\hline PTH604 & AML & 1 & 1 & 1 & 1 & 1 & 0 & 0 & 0 & 1 & 0 & 0 & 1 & 1 & 1 & 1 & 1 \\
\hline PTH693 & AML & 1 & 1 & 1 & 1 & 0 & 0 & 0 & 1 & 0 & 0 & 1 & 1 & 1 & 1 & 1 & 1 \\
\hline PTH711 & AML & 1 & 1 & 1 & 1 & 0 & 0 & 0 & 0 & 0 & 0 & 0 & 1 & 1 & 1 & 1 & 1 \\
\hline PTH7 18 & AML & 1 & 1 & 1 & 1 & 0 & 0 & 0 & 1 & 0 & 0 & 1 & 1 & 1 & 1 & 1 & 1 \\
\hline PTH724 & AML & 1 & 1 & 1 & 1 & 1 & 0 & 0 & 1 & 1 & 0 & 0 & 1 & 1 & 1 & 1 & 1 \\
\hline PTH788 & CML & 1 & 1 & 1 & 1 & 1 & 1 & 0 & 1 & 1 & 0 & 0 & 1 & 1 & 1 & 1 & 1 \\
\hline PTH 800 & AML & 1 & 1 & 1 & 1 & 0 & 0 & 0 & 0 & 0 & 0 & 0 & 1 & 1 & 1 & 1 & 1 \\
\hline PTP946 & ALL & 1 & 1 & 1 & 1 & 0 & 0 & 0 & 0 & 0 & 0 & 0 & 1 & 1 & 1 & 1 & 1 \\
\hline PTP097 & CML & 1 & 1 & 0 & 1 & 1 & 1 & 0 & 1 & 1 & 1 & 0 & 1 & 1 & 1 & 1 & 1 \\
\hline PTH224 & AML & 1 & 1 & 1 & 1 & 0 & 1 & 1 & 1 & 0 & 1 & 0 & 1 & 1 & 1 & 1 & 1 \\
\hline PTP251 & AML & 1 & 1 & 1 & 1 & 0 & 0 & 1 & 1 & 0 & 0 & 1 & 1 & 1 & 1 & 1 & 1 \\
\hline PTH268 & AML & 1 & 1 & 1 & 1 & 1 & 0 & 1 & 0 & 1 & 0 & 0 & 1 & 1 & 1 & 1 & 1 \\
\hline PTH3 16 & AML & 1 & 1 & 1 & 1 & 0 & 0 & 0 & 0 & 0 & 0 & 0 & 1 & 1 & 1 & 1 & 1 \\
\hline PTP 370 & CML & 1 & 1 & 1 & 1 & 0 & 0 & 0 & 0 & 0 & 0 & 0 & 1 & 1 & 1 & 1 & 1 \\
\hline
\end{tabular}


Histocompatibility Worskshop Conferences and Centre d'Etude du Polymorphisme Humain.

Analyzed samples were assigned as KIR genotype AA (when homozygosity for KIR haplotype A was detected), or as KIR genotype Bx when heterozygosity or homozygosity for KIR haplotype $B$ was typed (KIR genotypes AB and BB).

\section{Statistical analysis}

The occurrence of KIR genes in individuals (frequency $=$ F) was obtained by direct counting. Gene frequencies (GF) were calculated using the formula $G F=1-\sqrt{ }(1-F)$, being aware of the limitation in its ability to detect KIR genes present at low frequency. For analysis of the molecular polymorphism of the locus studied, the Arlequin software version 3.0 was used. Frequencies for specific genes or genotypes found in the patients group were compared with corresponding frequencies in general Macedonian population, that we have published previously [20]. Comparisons were done using the Fisher's two-sided exact test and Pearson's chisquared test. Risk ratios or crude odds ratios (OR) were calculated within 95\% confidence interval. Statistical significance was set to 0.05 .

\section{Results}

\section{KIR gene frequencies}

The KIR gene content and the diagnosis of the 63 Macedonian patients with haematological malignancies are shown in Table 1.

The frequencies of the 16 KIR genes (14 genes and 2 pseudogenes) determined in the 63 haematological patients along with the corresponding frequencies of the 214 healthy Macedonian controls are shown in Table 2. All 16 KIR genes were observed in the studied population and framework genes (KIR3DL3, KIR3DP1, KIR2DL4, and $K I R 3 D L 2)$ were present in all individuals, except for three patients who lacked the KIR3DL2 gene.

Comparison of KIR gene frequencies between the 63 patients and healthy Macedonians reveals statistically significant difference for the KIR3DL2 framework gene ( $F=1$ in the control group, and 0.95 in the patients group, $\mathrm{p}=0.001$ ).

\section{Genotype frequencies}

If any of the genes 2DL2, 2DL5, 3DS1, 2DS1,
2DS2, 2DS3, or 2DS5 was present; the genotype was considered as B. If none of these were present, genotype is considered as AA. We have not attempted to distinguish between AB and BB genotypes and called any of this Bx, nor have we numerated the individual Bx genotypes. Total of 28 different KIR genotypes have been determined in the 63 patients (Table 3). We have determined single AA genotype in 14 individuals from the patients group (20.5\%) which was comparable with the frequency of AA genotypes in control individuals (21.5\%, $\mathrm{P}=0.902)$. Statistically significant difference was found for the frequency of the Bx genotype 439, present in two patients and none of the healthy controls $(P=0.009)$, and also for the Bx genotype 3 (present in 5 patients and 4 controls, $\mathrm{P}=0.017$ ) (Table 3).

\section{Discussion}

There are no precise and reliable figures for incidence and survival rates for the different forms of haematological cancer even in highly developed Western European countries and USA. Although the health authorities and their bodies in different regions do publish descriptive statistics, there are many problems with this statistics, such as many cases are never reported to cancer registries, so the actual number of patients could be substantially higher than national figures suggest. Registration figures from United Kingdom for example, suggest around 50 new cases per population of 100,000 [21], but again, those working in the field believe that this number is considerably higher.

Having in mind the different results regarding the distribution of KIR genes reported in patients with certain forms of haematological cancers [14-17], we have analyzed the KIR genes distribution in Macedonian patients with haematological malignancies and compared them to healthy Macedonians. Although two recent studies reported higher frequency of activating KIR genes, such as KIR2DS1, KIR2DS3 and KIR2DS4 in patients with CML and ALL [22, 23], we couldn't reproduce these findings in our cohort. Our findings are thus in agreement with those reported from Babor et al, who also found very similar frequencies of KIR genes among haematological patients and healthy controls with European origin [24]. One significant difference that we have observed exclusively in our study was the one involving the frequency of the framework gene KIR3DL2 (Pearson's $\mathrm{P}=0.001$, after applying Yate's correction $\mathrm{P}=$ 0.012). However, comparison of frequencies obtained from larger (or META analysis) series would be needed 
Table 3: KIR locus haplogroups, genotypes ID and genotype frequency of Macedonian patients with haematological malignancies $(\mathrm{N}=63)$ and healthy Macedonians $(\mathrm{N}=214)$.

\begin{tabular}{|c|c|c|c|c|c|c|c|c|c|c|c|c|c|c|c|c|c|c|c|c|}
\hline $\begin{array}{l}\text { Haplo } \\
\text { group }\end{array}$ & $\begin{array}{l}\text { Geno } \\
\text { type } \\
\text { ID }\end{array}$ & $\begin{array}{c}K I R \\
3 D L 1\end{array}$ & $\begin{array}{c}K I R \\
2 D L 1\end{array}$ & $\begin{array}{c}K I R \\
2 D L 3\end{array}$ & $\begin{array}{c}K I R \\
2 D S 4\end{array}$ & $\begin{array}{c}K I R \\
2 D L 2\end{array}$ & $\begin{array}{c}K I R \\
2 D L 5\end{array}$ & $\begin{array}{c}K I R \\
3 D S 1\end{array}$ & $\begin{array}{c}K I R \\
2 D S 1\end{array}$ & $\begin{array}{c}K I R \\
2 D S 2\end{array}$ & $\begin{array}{c}K I R \\
2 D S 3\end{array}$ & $\begin{array}{c}K I R \\
2 D S 5\end{array}$ & $\begin{array}{c}K I R \\
2 D L 4\end{array}$ & $\begin{array}{c}K I R \\
3 D L 2\end{array}$ & $\begin{array}{c}K I R \\
3 D L 3\end{array}$ & $\begin{array}{c}K I R \\
2 D P 1\end{array}$ & $\begin{array}{c}K I R \\
3 D P 1\end{array}$ & $\begin{array}{l}\text { Malignancies } \\
\quad \text { № }(F)\end{array}$ & $\begin{array}{l}\text { Macedonians } \\
\quad \text { No }(F)\end{array}$ & $\begin{array}{c}\text { Pearson's } \\
\text { P }\end{array}$ \\
\hline AA & 1 & 1 & 1 & 1 & 1 & 0 & 0 & 0 & 0 & 0 & 0 & 0 & 1 & 1 & 1 & 1 & 1 & $14(0,206)$ & $46(0,215)$ & 0,902 \\
\hline $\mathrm{Bx}$ & 2 & 1 & 1 & 1 & 1 & 0 & 1 & 1 & 1 & 0 & 0 & 1 & 1 & 1 & 1 & 1 & 1 & $1(0,016)$ & $11(0,051)$ & 0,178 \\
\hline $\mathrm{Bx}$ & 3 & 1 & 1 & 1 & 1 & 1 & 1 & 1 & 1 & 1 & 0 & 1 & 1 & 1 & 1 & 1 & 1 & $5(0,079)$ & $4(0,019)$ & 0,017 \\
\hline $\mathrm{Bx}$ & 4 & 1 & 1 & 1 & 1 & 1 & 0 & 0 & 0 & 1 & 0 & 0 & 1 & 1 & 1 & 1 & 1 & $4(0,064)$ & $27(0,126)$ & 0,165 \\
\hline $\mathrm{Bx}$ & 5 & 1 & 1 & 1 & 1 & 1 & 1 & 0 & 0 & 1 & 1 & 0 & 1 & 1 & 1 & 1 & 1 & $1(0,016)$ & $8(0,037)$ & 0,397 \\
\hline $\mathrm{Bx}$ & 6 & 1 & 1 & 1 & 1 & 1 & 1 & 1 & 1 & 1 & 1 & 1 & 1 & 1 & 1 & 1 & 1 & $3(0,048)$ & $8(0,037)$ & 0,715 \\
\hline $\mathrm{Bx}$ & 7 & 1 & 1 & 1 & 1 & 1 & 1 & 1 & 1 & 1 & 1 & 0 & 1 & 1 & 1 & 1 & 1 & $1(0,016)$ & $6(0,028)$ & 0,589 \\
\hline $\mathrm{Bx}$ & 8 & 1 & 1 & 1 & 1 & 0 & 1 & 1 & 1 & 0 & 1 & 0 & 1 & 1 & 1 & 1 & 1 & $1(0,016)$ & $2(0.009)$ & 0,660 \\
\hline $\mathrm{Bx}$ & 9 & 1 & 1 & 1 & 1 & 1 & 1 & 0 & 1 & 1 & 0 & 1 & 1 & 1 & 1 & 1 & 1 & 0 & $5(0,023)$ & 0,221 \\
\hline $\mathrm{Bx}$ & 10 & 1 & 1 & 1 & 1 & 0 & 0 & 0 & 0 & 1 & 0 & 0 & 1 & 1 & 1 & 1 & 1 & $1(0,016)$ & $1(0,005)$ & 0,356 \\
\hline $\mathrm{Bx}$ & 11 & 1 & 1 & 1 & 1 & 1 & 1 & 0 & 1 & 1 & 1 & 0 & 1 & 1 & 1 & 1 & 1 & $3(0.048)$ & $6(0,028)$ & 0,445 \\
\hline $\mathrm{Bx}$ & 13 & 1 & 1 & 1 & 1 & 1 & 1 & 1 & 0 & 1 & 1 & 0 & 1 & 1 & 1 & 1 & 1 & 0 & $6(0,028)$ & 0,178 \\
\hline $\mathrm{Bx}$ & 14 & 1 & 1 & 1 & 1 & 0 & 0 & 1 & 0 & 0 & 0 & 0 & 1 & 1 & 1 & 1 & 1 & 0 & $3(0,014)$ & 0,345 \\
\hline $\mathrm{Bx}$ & 15 & 1 & 1 & 1 & 1 & 0 & 0 & 0 & 1 & 0 & 0 & 0 & 1 & 1 & 1 & 1 & 1 & $4(0,064)$ & $9(0,042)$ & 0,479 \\
\hline $\mathrm{Bx}$ & 16 & 1 & 1 & 1 & 1 & 0 & 0 & 1 & 1 & 0 & 0 & 0 & 1 & 1 & 1 & 1 & 1 & 0 & $1(0,005)$ & 0,587 \\
\hline $\mathrm{Bx}$ & 18 & 1 & 1 & 1 & 1 & 1 & 1 & 1 & 1 & 0 & 0 & 1 & 1 & 1 & 1 & 1 & 1 & 0 & $2(0,009)$ & 0,441 \\
\hline $\mathrm{Bx}$ & 19 & 1 & 1 & 1 & 1 & 1 & 0 & 0 & 0 & 0 & 0 & 0 & 1 & 1 & 1 & 1 & 1 & 0 & $1(0,005)$ & 0,587 \\
\hline $\mathrm{Bx}$ & 23 & 1 & 1 & 1 & 1 & 0 & 0 & 0 & 0 & 0 & 0 & 1 & 1 & 1 & 1 & 1 & 1 & 0 & $1(0,005)$ & 0,587 \\
\hline $\mathrm{Bx}$ & 28 & 1 & 1 & 1 & 1 & 0 & 1 & 1 & 1 & 0 & 1 & 1 & 1 & 1 & 1 & 1 & 1 & 0 & $1(0,005)$ & 0,587 \\
\hline $\mathrm{Bx}$ & 29 & 1 & 1 & 1 & 1 & 1 & 1 & 0 & 1 & 1 & 0 & 0 & 1 & 1 & 1 & 1 & 1 & $1(0,016)$ & 0 & 0,065 \\
\hline $\mathrm{Bx}$ & 33 & 1 & 1 & 1 & 1 & 0 & 1 & 1 & 1 & 0 & 0 & 0 & 1 & 1 & 1 & 1 & 1 & 0 & $1(0,005)$ & 0,587 \\
\hline $\mathrm{Bx}$ & 62 & 1 & 1 & 1 & 1 & 1 & 0 & 0 & 0 & 1 & 1 & 0 & 1 & 1 & 1 & 1 & 1 & 0 & $3(0,014)$ & 0,362 \\
\hline $\mathrm{Bx}$ & 63 & 1 & 1 & 1 & 1 & 1 & 0 & 1 & 1 & 1 & 0 & 1 & 1 & 1 & 1 & 1 & 1 & $1(0,016)$ & $2(0,009)$ & 0,660 \\
\hline $\mathrm{Bx}$ & 69 & 0 & 1 & 1 & 0 & 0 & 1 & 1 & 1 & 0 & 0 & 1 & 1 & 1 & 1 & 1 & 1 & 0 & $2(0,009)$ & 0,441 \\
\hline $\mathrm{Bx}$ & 70 & 0 & 1 & 1 & 0 & 1 & 1 & 1 & 1 & 1 & 1 & 1 & 1 & 1 & 1 & 1 & 1 & 0 & $2(0,009)$ & 0,441 \\
\hline $\mathrm{Bx}$ & 71 & 1 & 1 & 0 & 1 & 1 & 1 & 0 & 0 & 1 & 1 & 0 & 1 & 1 & 1 & 1 & 1 & 0 & $4(0,019)$ & 1 \\
\hline $\mathrm{Bx}$ & 72 & 1 & 0 & 0 & 1 & 1 & 0 & 0 & 0 & 1 & 0 & 0 & 1 & 1 & 1 & 0 & 1 & 0 & $1(0,005)$ & 0,587 \\
\hline $\mathrm{Bx}$ & 73 & 1 & 1 & 0 & 1 & 1 & 1 & 1 & 1 & 1 & 1 & 1 & 1 & 1 & 1 & 1 & 1 & 0 & $5(0,023)$ & 0,398 \\
\hline $\mathrm{Bx}$ & 76 & 1 & 0 & 0 & 1 & 1 & 1 & 1 & 1 & 1 & 0 & 1 & 1 & 1 & 1 & 0 & 1 & 0 & $2(0,009)$ & 0,441 \\
\hline $\mathrm{Bx}$ & 87 & 0 & 1 & 1 & 1 & 1 & 1 & 1 & 1 & 1 & 1 & 1 & 1 & 1 & 1 & 1 & 1 & 0 & $1(0,005)$ & 0,587 \\
\hline $\mathrm{Bx}$ & 90 & 1 & 1 & 0 & 1 & 1 & 1 & 1 & 1 & 1 & 1 & 0 & 1 & 1 & 1 & 1 & 1 & 0 & $2(0,009)$ & 0,441 \\
\hline $\mathrm{Bx}$ & 113 & 1 & 1 & 0 & 1 & 1 & 1 & 0 & 1 & 1 & 1 & 0 & 1 & 1 & 1 & 1 & 1 & $1(0.016)$ & $1(0,005)$ & 0,356 \\
\hline $\mathrm{Bx}$ & 159 & 0 & 1 & 1 & 0 & 1 & 1 & 1 & 1 & 1 & 1 & 0 & 1 & 1 & 1 & 1 & 1 & 0 & $1(0,005)$ & 0,587 \\
\hline $\mathrm{Bx}$ & 188 & 1 & 1 & 1 & 1 & 1 & 0 & 1 & 0 & 1 & 0 & 0 & 1 & 1 & 1 & 1 & 1 & $1(0.016)$ & $1(0,005)$ & 0,356 \\
\hline $\mathrm{Bx}$ & 192 & 1 & 1 & 1 & 1 & 1 & 0 & 0 & 1 & 1 & 0 & 0 & 1 & 1 & 1 & 1 & 1 & $1(0,016)$ & 0 & 0,065 \\
\hline $\mathrm{Bx}$ & 200 & 1 & 1 & 1 & 1 & 0 & 1 & 0 & 0 & 0 & 0 & 0 & 1 & 1 & 1 & 1 & 1 & 0 & $1(0,005)$ & 0,587 \\
\hline $\mathrm{Bx}$ & 202 & 1 & 1 & 1 & 1 & 1 & 0 & 0 & 1 & 1 & 0 & 1 & 1 & 1 & 1 & 1 & 1 & $3(0,048)$ & $3(0,014)$ & 0,107 \\
\hline $\mathrm{Bx}$ & 205 & 1 & 1 & 1 & 1 & 0 & 0 & 0 & 1 & 0 & 1 & 0 & 1 & 1 & 1 & 1 & 1 & 0 & $1(0,005)$ & 0,367 \\
\hline $\mathrm{Bx}$ & 233 & 1 & 1 & 1 & 1 & 1 & 0 & 1 & 1 & 1 & 1 & 0 & 1 & 1 & 1 & 1 & 1 & $1(0,016)$ & $5(0,023)$ & 0,716 \\
\hline $\mathrm{Bx}$ & 260 & 1 & 1 & 1 & 1 & 0 & 0 & 0 & 0 & 0 & 1 & 0 & 1 & 1 & 1 & 1 & 1 & 0 & $2(0,009)$ & 0,441 \\
\hline $\mathrm{Bx}$ & 268 & 1 & 1 & 1 & 1 & 1 & 0 & 0 & 0 & 1 & 1 & 1 & 1 & 1 & 1 & 1 & 1 & 0 & $1(0,005)$ & 0,587 \\
\hline $\mathrm{Bx}$ & 276 & 1 & 0 & 0 & 1 & 1 & 0 & 1 & 1 & 1 & 0 & 1 & 1 & 1 & 1 & 0 & 1 & $1(0,016)$ & $1(0,005)$ & 0,356 \\
\hline $\mathrm{Bx}$ & 294 & 0 & 1 & 1 & 0 & 1 & 1 & 1 & 1 & 0 & 1 & 1 & 1 & 1 & 1 & 1 & 1 & $1(0,016)$ & $1(0,005)$ & 0,356 \\
\hline $\mathrm{Bx}$ & 317 & 1 & 1 & 0 & 1 & 1 & 0 & 0 & 0 & 1 & 1 & 0 & 1 & 1 & 1 & 1 & 1 & 0 & $3(0,014)$ & 0,362 \\
\hline $\mathrm{Bx}$ & 318 & 0 & 1 & 0 & 0 & 1 & 0 & 1 & 1 & 1 & 1 & 1 & 1 & 1 & 1 & 1 & 1 & 0 & $1(0,005)$ & 0,587 \\
\hline $\mathrm{Bx}$ & 319 & 1 & 1 & 1 & 1 & 1 & 0 & 1 & 1 & 1 & 1 & 1 & 1 & 1 & 1 & 1 & 1 & $3(0,048)$ & $2(0,009)$ & 0,045 \\
\hline $\mathrm{Bx}$ & 331 & 1 & 1 & 1 & 0 & 0 & 1 & 1 & 1 & 0 & 1 & 0 & 1 & 1 & 1 & 1 & 1 & 0 & $\begin{array}{c}2-0,0) \\
0\end{array}$ & 0,098 \\
\hline $\mathrm{Bx}$ & 336 & 1 & 1 & 1 & 1 & 1 & 0 & 1 & 0 & 0 & 0 & 0 & 1 & 1 & 1 & 1 & 1 & 0 & $1(0,005)$ & 0,587 \\
\hline $\mathrm{Bx}$ & 359 & 1 & 0 & 1 & 1 & 0 & 1 & 1 & 1 & 0 & 0 & 1 & 1 & 1 & 1 & 1 & 1 & 0 & $1(0,005)$ & 0,587 \\
\hline $\mathrm{Bx}$ & 363 & 1 & 0 & 1 & 1 & 1 & 1 & 1 & 1 & 1 & 0 & 1 & 1 & 1 & 1 & 1 & 1 & 0 & $1(0,005)$ & 0,587 \\
\hline $\mathrm{Bx}$ & 370 & 1 & 1 & 1 & 1 & 1 & 0 & 0 & 1 & 1 & 0 & 1 & 1 & 1 & 1 & 1 & 1 & 0 & $3(0,014)$ & 0,362 \\
\hline $\mathrm{Bx}$ & 371 & 1 & 1 & 1 & 1 & 1 & 0 & 0 & 1 & 0 & 0 & 1 & 1 & 1 & 1 & 1 & 1 & 0 & $1(0,005)$ & 0,587 \\
\hline $\mathrm{Bx}$ & 372 & 1 & 1 & 1 & 1 & 0 & 0 & 1 & 1 & 0 & 1 & 0 & 1 & 1 & 1 & 1 & 1 & 0 & $1(0,005)$ & 0,587 \\
\hline $\mathrm{Bx}$ & 373 & 1 & 1 & 1 & 1 & 1 & 0 & 0 & 1 & 1 & 1 & 0 & 1 & 1 & 1 & 1 & 1 & $3(0.048)$ & $2(0,009)$ & 0,045 \\
\hline $\mathrm{Bx}$ & 374 & 1 & 0 & 1 & 0 & 1 & 1 & 1 & 1 & 1 & 1 & 0 & 1 & 1 & 1 & 1 & 1 & 0 & $1(0,005)$ & 0,587 \\
\hline $\mathrm{Bx}$ & 375 & 0 & 1 & 1 & 0 & 0 & 0 & 0 & 1 & 0 & 0 & 1 & 1 & 1 & 1 & 1 & 1 & 0 & $1(0,005)$ & 0,587 \\
\hline $\mathrm{Bx}$ & 376 & 0 & 0 & 0 & 1 & 1 & 0 & 0 & 1 & 1 & 1 & 0 & 1 & 1 & 1 & 1 & 1 & 0 & $1(0,005)$ & 0,587 \\
\hline $\mathrm{Bx}$ & 377 & 0 & 0 & 1 & 0 & 1 & 1 & 1 & 1 & 1 & 0 & 1 & 1 & 1 & 1 & 1 & 1 & 0 & $3(0,014)$ & 0,362 \\
\hline $\mathrm{Bx}$ & 378 & 1 & 1 & 0 & 1 & 1 & 0 & 1 & 0 & 1 & 0 & 0 & 1 & 1 & 1 & 0 & 0 & 0 & $1(0,005)$ & 0,587 \\
\hline $\mathrm{Bx}$ & 379 & 1 & 1 & 0 & 1 & 1 & 0 & 1 & 1 & 1 & 0 & 1 & 1 & 1 & 1 & 1 & 1 & 0 & $1(0,005)$ & 0,587 \\
\hline $\mathrm{Bx}$ & 380 & 1 & 0 & 1 & 1 & 1 & 1 & 0 & 0 & 1 & 0 & 0 & 1 & 1 & 1 & 1 & 1 & 0 & $1(0,005)$ & 0,587 \\
\hline $\mathrm{Bx}$ & 439 & 1 & 1 & 1 & 1 & 0 & 0 & 0 & 1 & 0 & 0 & 1 & 1 & 1 & 1 & 1 & 1 & $2(0,032)$ & 0 & 0,009 \\
\hline $\mathrm{Bx}$ & new & 0 & 1 & 1 & 1 & 0 & 1 & 1 & 1 & 1 & 1 & 0 & 1 & 1 & 1 & 1 & 1 & $1(0,016)$ & 0 & 0,065 \\
\hline $\mathrm{Bx}$ & new & 0 & 1 & 1 & 1 & 0 & 0 & 0 & 1 & 0 & 0 & 0 & 1 & 0 & 1 & 1 & 1 & $1(0,016)$ & 0 & 0,065 \\
\hline $\mathrm{Bx}$ & new & 1 & 1 & 0 & 1 & 1 & 0 & 1 & 0 & 1 & 1 & 0 & 1 & 1 & 1 & 1 & 1 & $1(0,016)$ & 0 & 0,065 \\
\hline $\mathrm{Bx}$ & new & 1 & 1 & 0 & 1 & 1 & 0 & 0 & 1 & 1 & 1 & 0 & 1 & 1 & 1 & 1 & 1 & $1(0,016)$ & 0 & 0,065 \\
\hline $\mathrm{Bx}$ & new & 1 & 1 & 1 & 1 & 1 & 0 & 0 & 1 & 1 & 0 & 0 & 1 & 1 & 1 & 1 & 1 & $1(0,016)$ & 0 & 0,065 \\
\hline
\end{tabular}

KIR Genotype [1=Positive, 0=negative]

in order to determine this association as an important protective one in the development of haematological cancers.

As for the comparison of KIR genotype frequencies, we didn't see any significantly different distribution of $A A$ and $B x$ genotypes between the two groups $(P=0.902)$. We have observed two statistically

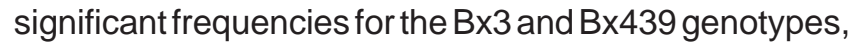
both more often present in the patients group $(P=0.019$ and $P=0.009$, respectively). However, the KIR gene content of the two genotypes is quit different, the former containing all the known KIR genes except KIR2DS3, and the later lacking 6 genes (both inhibitory and activating) (Table 3), we were not able to draw a conclusion that these might be prototypic KIR genotypes that would increase susceptibility for haematological cancer.

For many of the different forms of haematological cancers, the transplantation of haematopoietic stem cells from bone marrow or peripheral blood either from related or unrelated donors, has become widely recognized as the only curative treatment over the last 
three decades. Today, it is generally believed that donors containing at most one activating KIR gene should be avoided if possible, because they could more often lead to severe acute GVHD [25 - 28]. Our next goal would be to analyze the distribution of KIR genes and the success rate in transplanted pairs of patients with haematological cancer and their corresponding bone marrow donor.

We found that KIR gene frequencies between the total 24 donors and healthy Macedonians reveals statistically significant difference for KIR2DS1 ( $F=0.481$ in the controls group, and 0.76 in the patients group, $p=0.004)$. This significance is even higher when the frequency of KIR2DS1 in controls is compared with the frequency in donors from pairs with $\mathrm{GVHD}(\mathrm{F}=0.923, \mathrm{P}=$ 0.002 ). Another significant difference was observed for the frequency of the full-length allele of KIR2DS4*001002 , present in $25.2 \%$ of the control individuals, but in as much as $81.8 \%$ of the recipients of $\mathrm{HSC}(\mathrm{P}=0.0005)$ and suggest that the KIR2DS4*001/002 might be a predisposing factor for severe GVHD in sibling HSCT [29].

We investigated the role of KIR genes in some viral infections in the Republic of Macedonia. Comparison of KIR gene frequencies between critically ill H1N1/09 Macedonian patients and healthy subjects reveals statistically significant difference for frequency of KIR2DL1 ( $F=1$ in the patients group, and 0.94 in the control group, $p=0.045)$. There is evident predominance of KIR activating genes in the group of patients with severe disease, compared to the healthy controls [30]. Recently we published that comparison of KIR frequencies between Macedonian patients with West Nile virus infection and healthy Macedonian population reveals several significant differences in the inhibitory group (KIR2DL2), and in the non inhibitory group (KIR2DS1, KIR2DS2, KIR2DS5, and KIR3DS1). The single most frequent genotypes in the Bx group were genotypes ID71 and ID89 with statistically significant difference compared to healthy Macedonians. Our results suggest that specific KIR genotypes could be connected with West Nile virus infection [31].

In conclusion, our results address the distribution of the KIR genes and genotypes in Macedonian patients with haematological cancers. Although we have not determined a certain KIR gene and/or genotype as either predisposing, or protecting factor for haematological malignancy in patients from Republic of Macedonia, we believe that our results could be useful in further (perhaps META) analysis, involving larger series of patients and targeted at the ligands of the KIRs that might suggest involvement of certain combinations of these genes in the development of haematological cancers.

\section{Acknowledgement}

The authors thank Elena Cvetkovska (Institute of Immunobiology and Human Genetics, Faculty of Medicine, Ss Cyril and Methodius University, Skopje, Republic of Macedonia) for isolation of genomic DNA, and taking care of the Macedonian Human DNA Bank (http://www.hdnamkd.org.mk).

\section{References}

1. Lotze MA. Thomson - Natural Killer Cells: Basic Science and Clinical Application. $1^{\text {st }}$ Ed. Academic Press, London, UK, 2010.

2. French AR, Yokoyama WM. Natural killer cells and viral infections. Curr Opin Immunol. 2003; 15:45-51.

3. Diefenbach $\mathrm{A}$, Raulet $\mathrm{DH}$. The innate immune response to tumors and its role in the induction of T-cell immunity. Immunol Rev. 2002; 188:9-21.

4. Liu WR, Kim J, Nwankwo C, Ashworth LK, Arm JP. Genomic organization of the human leukocyte immunoglobulin-like receptors within the leukocyte receptor complex on chromosome 19q13.4. Immunogenetics. 200; 51(8-9), 65969.

5. Hsu KC, Chida S, Geraghty DE, Dupont B. The killer cell immunoglobulin-like receptor (KIR) genomic region: geneorder, haplotypes and allelic polymorphism. Immunological Reviews. 2002;190:40-52.

6. Uhrberg M, Valiante NM, Shum BP, Shilling HG, LienertWeidenbach K, Corliss B, Tyan D, Lanier LL, Parham P. Human diversity in killer cell inhibitory receptor genes. Immunity. 1997;7(6):753-63.

7. Gonzalez A, McErlean C, Meenagh A, Shovlin T, Middleton D. Killer cell immunoglobulin-like receptor allele discrimination by high-resolution melting. Hum Immunol. 2009;70(10):85863.

8. Middleton D, Gonzelez F. The extensive polymorphism of KIR genes. Immunology. 2010;129(1):8-19.

9. Trowsdale J. Genetic and functional relationships between MHC and NK receptor genes. Immunity. 2001;15:363-74.

10. Ljunggren HG, Kärre K. In search of the 'missing self': MHC molecules and NK cell recognition. Immunol Today. 1990; 11:237-44.

11. Demanet C, Mulder A, Deneys V, Worsham MJ, Maes P, 
Claas FH, et al. Down-regulation of HLA-A and HLA-Bw6, but not HLA-Bw4, allospecificities in leukemic cells: an escape mechanism from CTL and NK attack? Blood. 2004; 103:312230 .

12. Brouwer RE, van der Heiden P, Schreuder GM, Mulder A, Datema G, Anholts JD, et al. Loss or downregulation of HLA class I expression at the allelic level in acute leukemia is infrequent but functionally relevant, and can be restored by interferon. Hum Immunol. 2002; 63:200-10.

13. Vollmer M, Li L, Schmitt A, Greiner J, Reinhardt P, Ringhoffer $M$, et al. Expression of human leucocyte antigens and co-stimulatory molecules on blasts of patients with acute myeloid leukaemia. Br J Haematol. 2003; 120:1000-8.

14. van der Meer A, Schaap NP, Schattenberg AV, van Cranenbroek B, Tijssen $\mathrm{HJ}$, Joosten I. Mol Immunol. 2008;45(13):3631-8.

15. Tajik N, Shahsavar F, Nasiri M, Radjabzadeh MF. Int J Immunogenet. 2010; 37(3):159-68.

16. Symons HJ, Leffell MS, Rossiter ND, Zahurak M, Jones RJ, Fuchs EJ. Biol Blood Marrow Transplant. 2010;16(4):53342.

17. Babor F, Manser A, Schönberg K, Enczmann J, Borkhardt A, Meisel R, Uhrberg M. Lack of association between KIR genes and acute lymphoblastic leukemia in children. Blood. 2012;120(13): 2770-2

18. Towner P. Purification of DNA. Essential Molecular Biology T. A. Brown. Oxford, Oxford University Press, 1995:47-54.

19. Spiroski M, Arsov T, Petlichkovski A, Strezova A, Trajkov D, Efinska-Mladenovska O, Zaharieva E. Case Study: Macedonian Human DNA Bank (hDNAMKD) as a source for public health Genetics. Health Determinants in the Scope of New Public Health. B. G. Georgieva L. Sofia, Hans Jacobs Company, 2005:33-44.

20. Djulejic E, Petlichkovski A, Trajkov D, Hristomanova S, Middleton D, Spiroski M. Distribution of killer cell immunoglobulinlike receptors in the Macedonian population. Hum Immunol. 2010;71(3):281-8.

21. National Institute for Clinical Excellence, Guidance on Cancer Services. Improving Outcomes in Haematological Cancers, the Manual. London, MidCity Place, October 2003.

22. Zhang Y, Wang B, Ye S, et al. Killer cell immunoglobulinlike receptor gene polymorphisms in patients with leukemia: possible association with susceptibility to the disease. Leukemia Res. 2010;34(1):55-58

23. Almalte Z, Samarani S, Iannello A, Debbeche O, Duval M, Infante-Rivard C, Amre DK, Sinnett D, Ahmad A. Novel associations between activating killer-cell immunoglobulin-like receptorgenes and childhood leukemia. Blood. 2011;118(5):1323-8.

24. Babor F, Manser A, Schönberg K, Enczmann J, Borkhardt A, Meisel R, Uhrberg M. Lack of association between KIR genes and acute lymphoblastic leukemia in children. Blood. 2012;120(13):2770-2; author reply 2772.

25. Moretta L, Locatelli F, Pende D, Marcenaro E, Mingari MC, Moretta A. Killer Ig-like receptor-mediated control of natural killer cell alloreactivity in haploidentical hematopoietic stem cell transplantation. Blood. 2011; 117:764-771.

26. Ljungman $P$, Bregni $M$, Brune $M$, Cornelissen J, de Witte T, Dini G et al. Allogeneic and autologous transplantation for haematological diseases, solid tumours and immune disorders: current practice in Europe 2009. Bone Marrow Transplant. 2010;45(2):219-34.

27. Hamilton BK, Copelan EA. Concise review: the role of hematopoietic stem cell transplantation in the treatment of acute myeloid leukemia. Stem Cells. 2012;30(8):1581-6.

28. Mohty M, Apperley JF. Long-term physiological side effects after allogeneic bone marrow transplantation. Hematology Am Soc Hematol Educ Program. 2010;2010:229-36.

29. Petlichkovski A, Stojanoski Z, Djulejic E, Georgievski B, Spiroski M. Association of Killer Cell Immunoglobulin-Like Receptor Genes with the Graft versus Host Disease after Related Haematopoietic Stem Cell Transplantation in Patients with Haematological Malignancies from Republic of Macedonia. Maced J Med Sci. 2012; 5(4):404-410.

30. Petlichkovski A, Milenkovic Z, Djulejic E, Jefremovska B, Babacic H, Ivanovski LJ, Kondova Topuzovska I, Spasovska K, Spiroski M. Association of Killer Cell Immunoglobulin-Like Receptor Genes with Pandemic Influenza A (H1N1)pdm09 Infection in Critically III Macedonian Patients. Maced J Med Sci. 2012; 5(3):288-295.

31. Spiroski M, Milenkovic Z, Petlichkovski A, Ivanovski L, Topuzovska IK, Djulejic E. Killer Cell Immunoglobulin-Like Receptor Genes in Four Human West Nile Virus Infections Reported 2011 in the Republic of Macedonia. Hum Immunol. 2012 Dec 5. pii: S0198-8859(12)00622-2. doi: 10.1016/ j.humimm.2012.11.015. 